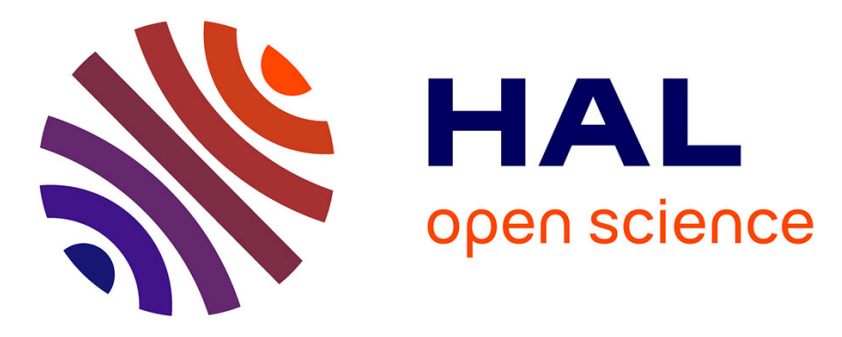

\title{
Coherent-stable scatterers detection in SAR multi-interferograms: feature fuzzy fusion in Alpine glacier geophysical context
}

Gabriel Vasile, Emmanuel Trouvé, Lionel Valet, Jean-Marie Nicolas, Lionel Bombrun, Michel Gay, Ivan Petillot, Philippe Bolon, Vasile Buzuloiu

\section{To cite this version:}

Gabriel Vasile, Emmanuel Trouvé, Lionel Valet, Jean-Marie Nicolas, Lionel Bombrun, et al.. Coherentstable scatterers detection in SAR multi-interferograms: feature fuzzy fusion in Alpine glacier geophysical context. IGARSS 2007 - IEEE International Geoscience and Remote Sensing Symposium, Jul 2007, Barcelone, Spain. hal-00144076

\section{HAL Id: hal-00144076 \\ https://hal.science/hal-00144076}

Submitted on 30 Apr 2007

HAL is a multi-disciplinary open access archive for the deposit and dissemination of scientific research documents, whether they are published or not. The documents may come from teaching and research institutions in France or abroad, or from public or private research centers.
L'archive ouverte pluridisciplinaire HAL, est destinée au dépôt et à la diffusion de documents scientifiques de niveau recherche, publiés ou non, émanant des établissements d'enseignement et de recherche français ou étrangers, des laboratoires publics ou privés. 


\title{
Coherent-stable scatterers detection in SAR multi-interferograms: feature fuzzy fusion in Alpine glacier geophysical context
}

\author{
G. VASILE ${ }^{* \dagger \S}$, E. TROUVÉ*†, L. VALET* ${ }^{*}$, J.-M. NICOLAS ${ }^{\ddagger}$, \\ L. BOMBRUN ${ }^{\dagger}$, M. GAY ${ }^{\dagger}$, I. PETILLOT* ${ }^{*}$, Ph. BOLON* and V. BUZULOIU ${ }^{\S}$ \\ * Université de Savoie, LISTIC - BP 806 - F-74016 Annecy Cedex, FRANCE \\ Tel: +33 450096548 - \{g.vasile|trouve|lionel.valet|ivan.petillot|bolon\}@univ-savoie.fr \\ $\dagger$ INP Grenoble, GIPSA-lab, CNRS - BP 46 - 38402 Saint-Martin-d'Hères, FRANCE \\ Tel: +33 476826256 - \{lionel.bombrun|michel.gay\}@lis.ingg.fr \\ $\ddagger$ GET Télécom Paris, LTCI, CNRS - 46, Rue Barrault - 75013 Paris, FRANCE - Tel: +33 145818129 - nicolas@tsi.enst.fr \\ $\S$ Univ. Politehnica Bucuresti, LAPI - Bd. Iuliu Maniu, Nr. 1-3, Bucharest, ROMANIA - Tel: +4021 4024683 - buzuloiu@alpha.imag.pub.ro
}

\begin{abstract}
SAR interferometry (InSAR) performs two acquisitions (spatially separated by the baseline) of the signal back-scattered by the resolution cell which contains height and/or displacement information. Repeat pass spaceborne interferometry provides multi-interferograms which can be used to extract such information either by combining the multi-temporal results of conventional interferometry or by a different approach based on specific targets: the coherent stable scatterers (CSS). In this paper a two-step approach is proposed to obtain specific features from multi-temporal InSAR data sets. The first step consists in extracting image attributes related to the useful information. The second step consists in merging the attributes using an interactive fuzzy fusion technique. The interactive fuzzy fusion is proposed to provide end-users with a simple and easily understandable tool for tuning the detection results. The method is applied on a data set of five co-registered ERS 1/2 tandems from the French Alps (the Mont-Blanc region), including two temperate glaciers: the Argentière and the Mer-de-glace. The results illustrate how the end-user can combine the proposed attributes to detect the presence of CSS or distributed stable scatterers usefull for multi-temporal analysis.
\end{abstract}

\section{INTRODUCTION}

Synthetic Aperture Radar interferometry (InSAR) has the potential to measure temperate glacier displacement with a large coverage compared to sparse terrestrial ground measurements [1]. Depending on the surface cover (rocks, snow or ice), the scattering properties may change with time and induce the decrease of interferogram coherence i.e. temporal decorrelation. More recent techniques have been proposed to select in an interferogram the pixels which exhibit less decorrelation than others in order to reduce such effects within conventional InSAR processing. Several discriminant parameters are used to describe these coherent or stable scatterers: either within one SAR acquisition (internal coherence [2], mutual information) or in a large number of interferograms [3].

In this paper a two-step approach is proposed to obtain simultaneously spatial and temporal information from multi-temporal InSAR data sets. The first step consists in extracting image attributes related to the useful information. The second step consists in merging the attributes using a interactive fuzzy fusion technique. The interactive fuzzy fusion is proposed to provide end-users with a simple and easily understandable tool for tuning the detection results. The interactive aspect allows the integration of prior information, expert knowledge, and/or new available data. The symbolic aspect is about the type of effective fusion of the data: in opposition of the numerical way where the values are directly merged by numerical operators, in a symbolic way a semantic description of the data is made and the fusion is realized at the symbol level which is close to the end user reasoning.

The proposed method is illustrated on a data set of five corregistered ERS 1/2 InSAR tandems from the French Alps (the Mont-Blanc region), including two temperate glaciers: the Argentière and the Mer-de-glace. The estimated attributes are:

- a new attribute to characterise the interferometric coherence temporal stability, the "multi-InSAR coherence", which combines the multi-temporal coherences estimated by IDAN technique [4].

- an attribute which characterizes the target coherence in each interferogram, the "sub-band coherence" [2],

- a texture multi-temporal feature derived from the second kind statistics (the 3rd order log-cumulants) which characterize the presence of coherent targets from their intensity in the time series [5],

The fuzzy fusion of such attributes provides information on the preservation of the interferometric coherence over the studied glaciers for velocity measurements, both on pointwise and distributed targets, usually called coherent or non-coherent targets according to the length of the antenna aperture where the target back-scattered signal remains coherent. Moreover, possible candidates for coherent and stable scatterers (CSS) are obtained for further CSS processing [3].

\section{ATtRIBUte ESTIMATION}

The first step of the proposed approach consists in developing attributes which reveal the presence of specific features in the repeat pass multi-temporal series of interferometric SAR images.

\section{A. Multi-InSAR coherence}

In multi-InSAR processing, it is important to have a good description of both scattering properties and temporal evolution of the target area, over the whole dataset.

One parameter for characterizing the stable scatterers within multiple interferograms is proposed, namely the 
"multi-InSAR coherence" $C_{v}$. This parameter is estimated using intensity-driven Adaptive-Neighborhood $A N$ [4] technique:

$$
C_{v}=\frac{\sum_{k=1}^{N} \sum_{i \in A N} z_{i_{k}}^{M} z_{i_{k}}^{S^{*}}}{\sqrt{\sum_{k=1}^{N} \sum_{i \in A N}\left|z_{i_{k}}^{M}\right|^{2} \cdot \sum_{k=1}^{N} \sum_{i \in A N}\left|z_{i_{k}}^{S}\right|^{2}}}
$$

where $N$ is the number of available InSAR couples, $z^{M}$ and $z^{S}$ are the master and slave SLC images, respectively. In Eq. (1), it is important to notice that the local deterministic component of the phase signal $\phi$ should be flattened when estimating the complex correlation within the 3D adaptive neighborhood. In this paper the adaptive HR-LR model of local frequencies $\left(f_{x}, f_{y}\right)$ [6] is used for the phase flattening:

$\phi_{f l}(k, l)=\phi(k, l)-2 \pi\left[(k-m) f_{x}+(l-n) f_{y}\right]-\phi(m, n)$,

where $(m, n)$ is the position of the central pixel within the interferogram and $(k, l) \in A N$ belong to the estimation sliding window.

\section{B. Sub-band coherence}

One parameter for characterizing the coherent scatterers within one interferogram is the "sub-band InSAR coherence" $C_{s u b}$. Firstly, the interferometric pair is decomposed in $B$ sub-bands as in [2]. Then, this 3D coherence is estimated within a spatial neighborhood $W$ as follows:

$$
C_{s u b}=\frac{\sum_{j=1}^{B} \sum_{i \in W} s_{i_{j}}^{M} s_{i_{j}}^{S^{*}}}{\sqrt{\sum_{j=1}^{B} \sum_{i \in W}\left|s_{i_{j}}^{M}\right|^{2} \cdot \sum_{j=1}^{B} \sum_{i \in W}\left|s_{i_{j}}^{S}\right|^{2}}}
$$

where $s^{M}$ and $s^{S}$ are the master and slave sub-bands, respectively.

\section{3rd Order Log-cumulant}

A different way to detect the strong intensities of CSS in multi-temporal data consists in estimating texture parameters in spatio-temporal windows made of the pixel of a small spatial neighborhood at the different dates.

The second kind statistics are well suited to estimate the parameters of the probability density functions defined on $\mathbf{R}^{+}$, such as the Gamma distributions for speckle intensity images. The second and third order log-cumulants have been proposed to reveal spatial features and temporal changes [5]. In homogeneous areas, the third order logcumulant $\tilde{\kappa}_{3}$ is close to the theoretical value $\Psi^{\prime \prime}(L)$ where $\Psi^{\prime \prime}$ is the second derivative of the Digamma function and $L$ the number of looks. In the presence of heterogeneous features such as strong intensity CSS, $\tilde{\kappa}_{3}$ values increase because of the heavy tail of the intensity distribution. It provides a complementary attribute to reaveal potential CSS from the multi-temporal intensity information.

\section{INTERACTIVE FUZZY FUSION SYSTEM}

The second step of the proposed approach combines the information extracted from the SAR images. Expert knowledge is introduced by using an interactive symbolic fuzzy fusion (ISFF) method [7]. The interactive and symbolic aspects are the main keys of the method detailed hereafter.

\section{A. Knowledge representation}

The attributes to be fused are described by the experts by means of several symbols (words). These symbols are related to the attribute numerical values through the membership functions. For each attribute $A_{i}, i \in\left[1, N_{A}\right]$, where $N_{A}$ is the number of attributes, a symbolic description is a set of $s_{i}$ words denoted by:

$$
\mathcal{L}_{i}=\left\{L_{i, 1}, L_{i, 2}, \ldots, L_{i, s_{i}}\right\}
$$

The membership functions are set by the system with equidistant modal values, but they can be adjusted later by the user (Section III-C).

The attributes represented by sets of symbols are aggregated according to the expert knowledge. This knowledge is modeled using the fuzzy subset theory, the experts being able to express their reasoning in a linguistic way by means of IF-THEN rules. A general rule example for the description of an output class is:

$$
\begin{gathered}
\text { IF } A_{1} \text { is } L_{1, j} \text { AND } A_{2} \text { is } L_{2, j^{\prime}} \text { AND } \ldots \\
\text { THEN pixel BELONGS TO } \text { out }_{k} .
\end{gathered}
$$

where $A_{i}, i \in\left[1, N_{A}\right]$ are the attributes, $L_{i, j}, j \in\left[1, s_{i}\right]$ the symbols that describe these attributes, and out $t_{k}$ is an output class, $k \in\left[1, N_{C}\right]$, where $N_{C}$ is the number of classes.

The fuzzy subset theory is an adequate tool to encode such linguistic knowledge [8]. In this theory, a rule-base is used to represent all the rules. In a three-input fusion case, this rule-base can be described in the three-dimensional (3D) attribute domain where each sub-cube represents a rule for a desired output class.

\section{B. Fusion principle/aggregation step}

The numerical input attributes are processed according to the membership functions in order to obtain a linguistic description. The aggregation of the linguistic descriptions is then performed by Zadeh's compositional rule of inference in order to obtain at each pixel the membership degree $\mu_{k}$ to the output class $k$ :

$$
\begin{array}{r}
\mu_{k}\left(a_{1}, a_{2}, \ldots\right)=\perp_{L_{1 j}, L_{2 j^{\prime}}, \ldots \in \mathcal{L}_{1}, \mathcal{L}_{2}, \ldots} \\
\top\left(\top\left(\mu_{D\left(a_{1}\right)}\left(L_{1 j}\right), \mu_{D\left(a_{2}\right)}\left(L_{2 j^{\prime}}\right), \ldots\right), \mu_{R}\left(L_{1 j}, L_{2 j^{\prime}}, \ldots k\right)\right)
\end{array}
$$

where $\left(a_{1}, \ldots\right)$ are the values taken by the attributes $A_{1}, \ldots$ at the current pixel. The term $\mu_{R}\left(L_{1 j}, \ldots, k\right)$ is equal to 1 if the corresponding rule is present in the rule-base and 0 otherwise. The fuzzy operators involved in this formula are chosen to obtain a system behavior close to the behavior of experts when they interpret images [7]. The selected operators are:

- $\top(a, b)=a * b$

- $\perp(a, b)=\min (a+b, 1)$

Finally, the decision step consists in classifying the considered image pixels into output classes according to the greatest output membership degree $\mu_{k}$. 


\section{Graphic user interface}

The proposed approach involves experts in the fusion of the attributes. The experts' role consists in initializing the fusion system thanks to their experience. They give the rules which define the sought-after classes and they provide the membership functions in order to adjust the meanings of the words used in the rules. These parameters must be set in an interactive way, in order to allow them to improve the detection. The other steps of the fuzzy method (the inference rule and the combination/projection operator) are automatic: the end users are not expected to interact in these steps which require specific knowledge in the fuzzy subset theory.

A Graphic User Interface (GUI) has been developed to enable an interactive introduction of the expert knowledge into the fusion of three attributes. The GUI is composed of a 3D space where each axis corresponds to an attribute. The linguistic parameters and the rules are easily set by experts directly in the representation cube. For each rule, the corresponding sub-cube can be stretched and the membership functions which describe the symbols involved in the rule are automatically adjusted in the same 3D space. In order to help experts in this adjustment, different sets of points corresponding to reference regions (pointed out by experts or given by masks), can be drawn in the attribute space. The objective is then to stretch the sub-cubes in order to isolate the different sets of points as precisely as possible. Then the system is ready to perform the fusion. The fuzzy results can be observed as images of the membership degrees to the output classes. Crisp results are obtained either interactively by thresholding one of the membership degrees or automatically by selecting the output class which obtain the highest degree.

\section{RESULTS AND DISCUSSION}

The proposed method has been developed to deal with the high mountainous relief present in the Chamonix Mont-Blanc test site which is located in the Alps, near the borders between France, Italy and Switzerland $\left(45^{\circ} 50^{\prime} \mathrm{N}\right.$, $6^{\circ} 51^{\prime} \mathrm{E}$ ). It includes the Aiguille-Verte (4122 $\mathrm{m} \mathrm{ASL}$ ), the Chamonix valley (1000 $\mathrm{m} \mathrm{ASL}$ ) and two instrumented glaciers: the Mer-de-Glace and the Argentière.

In this paper, 10 SAR images from ERS 1 and ERS 2 have been processed. This data set presented in Tab. I corresponds to 1-day tandem couples acquired in descending passes. After obtaining the SLC images, the five tandem interferograms were produced with an initial $2 \times 10$ complex multi-looking. Fig. 1 presents a color composition of three SAR amplitudes and coherences at three different months: October, January and March. One can notice that in Fig. 1, the gray regions correspond to non-changing features, while the colored regions exhibit an important change in radiometry.

After coarse corregistration of the five interferograms, the third order log-cumulant $\tilde{\kappa}_{3}$ of the SAR amplitudes is computed within a $3 \times 3 \times 10$ window (Fig. 2 -(a)). This parameter provides information on the stability of strong scatterers.
TABLE I

ERS SAR RAW DATA ACQUIRED OVER MONT-BLANC AREA WITH THE FRAME NUMBER 2673 AND $B$ : BASELINES (PARALLEL AND PERPENDICULAR TO THE RADAR LINE OF SIGHT).

\begin{tabular}{||c|c|c|c|}
\hline \hline Dates & Orbites & $B_{\|}(\mathbf{m})$ & $B_{\perp}(\mathbf{m})$ \\
\hline \hline $95.07 .09 / 95.07 .10$ & $20823 / 01150$ & -4 & 29 \\
\hline $95.10 .22 / 95.10 .23$ & $22326 / 02653$ & -44 & -107 \\
\hline $95.12 .31 / 96.01 .01$ & $23328 / 03655$ & 79 & 208 \\
\hline $96.03 .10 / 96.03 .11$ & $24330 / 04657$ & 26 & 9 \\
\hline $96.04 .14 / 96.04 .15$ & $24831 / 05158$ & 39 & 93 \\
\hline \hline
\end{tabular}

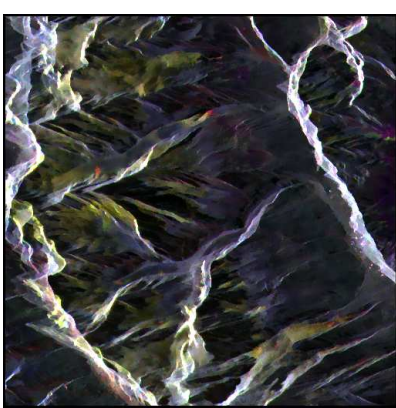

(a)

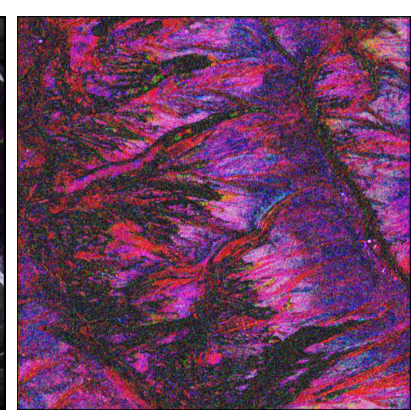

(b)
Fig. 1. Chamonix-Mont Blanc $[512 \times 512$ pixels]: (a) color composition of IDAN filtered SAR amplitudes (Red=1996/03/11, Green=1996/01/01, Blue $=1995 / 10 / 23$ ), (b) color composition of IDAN estimated SAR coherences $($ Red=1996/03/11, Green=1996/01/01, Blue=1995/10/23).

For the sub-band coherence attribute, each SLC image is decomposed into 5 sub-bands with $10 \%$ overlapping and Hamming weighting. The resulting sub-band coherences $C_{s u b}$ are obtained according to Eq. 3 with a $3 \times 3$ boxcar window. Then, the sub-band coherences obtained for the different InSAR couples are combined (Fig. 2(b)): as for the CSS detection the objective is to have an important coherence level in all coherence maps, the aggregation is performed by using the minimum operator:

$$
C_{\text {sub }}^{\min }=\min _{j \in\{1, . ., B\}} C_{s u b_{j}} .
$$

For the multi-InSAR coherence attribute, each 20-look interferogram is filtered independently by using IDAN technique [4] and compensating the deterministic phase component [6]. The multi-InSAR coherence illustrated in Fig. 2-(c) is computed according to Eq. 1.

Once the space defined by the three attributes from Fig. 2 is built, the 3D histogram is computed Fig. 3-(a). As the third order log-cumulant provides information on the strong scatterers within the SAR amplitude images, its distribution presents a main lobe in the vicinity of $\tilde{\kappa}_{3}=$ $\boldsymbol{\Psi}^{\prime \prime}(20)$ and outliers corresponding to strong scatterers. This induces a non-uniform distribution of the words employed in the fuzzy fusion step. After visualizing the 3D histogram, the end-user must tune the partition of the attribute space, assuring enough word sampling in each cloud of points.

The final step, before starting the fusion is to assign the set of rules corresponding to the desired classes. This can be performed by:

- expert (a priori) knowledge on the behaviour of the attributes. For example, if the sub-band coherence, 


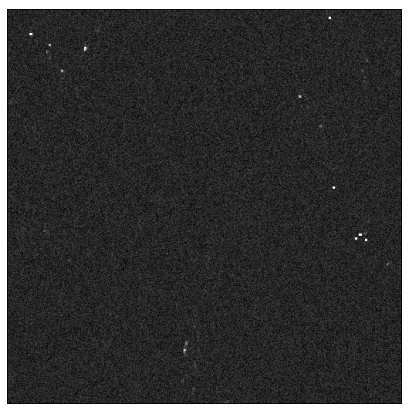

(a)

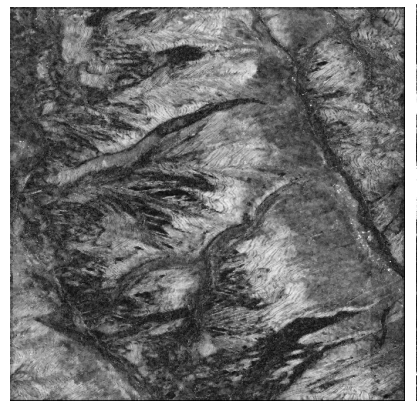

(c)

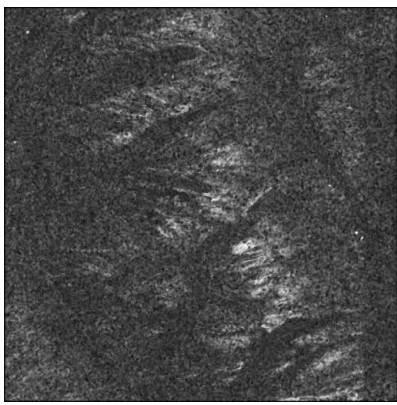

(b)

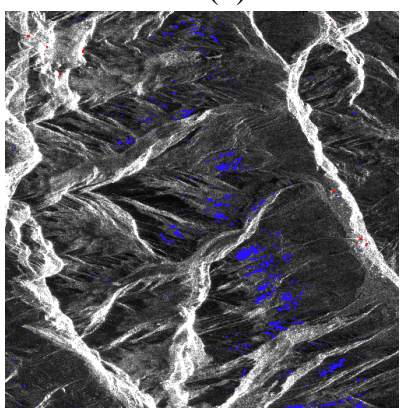

(d)
Fig. 2. Multi-InSAR attributes and fuzzy fusion results $[512 \times 512$ pixels]: (a) $\tilde{\kappa}_{3}$ third order log-cumulant $(3 \times 3 \times 5$ boxcar $)$, (b) $C_{\text {min }}^{\text {min }}$ minimum of the five coherences $(3 \times 3$ boxcar $),\left(\right.$ c) $C_{v}$ IDAN multiInSAR coherence, $(\mathrm{d})$ detection result $(\mathrm{Red}=\mathrm{CSS}$ and Blue $=$ distributed stable scatterers).

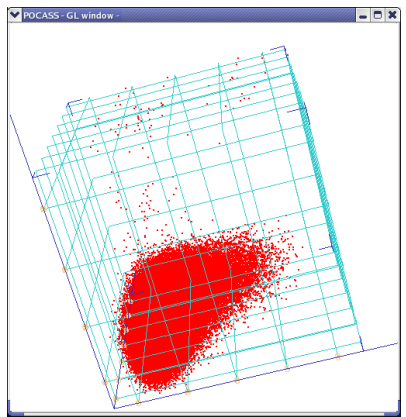

(a)

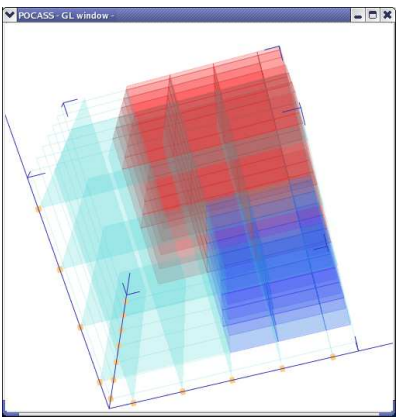

(b)
Fig. 3. 3D histogram (a) of the Multi-InSAR attributes from Fig. 2. The mesh within the attribute space corresponds to the interactive tuning of the words (b) used in the fuzzy fusion: horizontal axes multi-InSAR coherence (left to right) vs. minimum sub-band coherence (front to back); vertical axis $-\tilde{\kappa}_{3}$ ).

the multi-InSAR coherence and the third order logcumulants are large, then the output class is CSS (rules illustrated in red in Fig. 3-(b));

- visualizing clouds of points corresponding to different region of interests which are candidates for the desired classes. This regions of interest can be selected on either one of the attributes or on another image easier to interpret (ex: one of the SAR amplitude images).

The detection result is presented in Fig. 2-(d). The class marked in red represents the CSS points. As the obtained CSS points are only on non-moving areas (mainly in the Chamonix valley), CSSInSAR processing [3] for measuring glacier velocity is not applicable with ERS data on the studied glaciers. This behaviour is confirmed also over the distributed targets: only the rocky slopes are detected as they are not affected by the seasonal temperature variations. Since the ice/snow areas are rejected, the PSInSAR [9] analysis for glacier velocity measurements is also not applicable with ERS tandem data. Accordingly, both alternative techniques to conventional InSAR can not be used with annual interferogram stacking covering the summer season.

\section{CONCLUSION}

In this paper, a tunable fuzzy fusion system is proposed for the detection of specific features from multitemporal InSAR data sets: either distibuted stable scatterers with sufficient interferometric coherence for PSInSAR measurements, or CSSInSAR for displacement measurement based on specific targets. The nature of the application requires interaction between the intrinsic properties of SAR scatterers and qualitative information brought by experts. The proposed fusion system is designed to combine different sources of information by means of a fuzzy symbolic set of rules, the behavior of which is close to that of the end-user. The example presented on the Mont-Blanc temperate glaciers shows encouraging results in combining qualitative geophysical knowledge with objective parameters for detection-classification purposes: coherent stable scatterers and distributed stable scatterers selection. In perspective, it will be interesting to use such approaches in the analysis of future POL-InSAR data acquired over Alpine glaciers.

\section{ACKNOWLEDGMENTS}

This work was supported by the French national project ACIMEGATOR. The authors wish to thank to the European Space Agency for providing ERS SAR data through the Category 1 proposal No.3525.

\section{REFERENCES}

[1] E. Trouvé, G. Vasile, M. Gay, L. Bombrun, P. Grussenmeyer, T. Landes, J.M. Nicolas, Ph. Bolon, I. Petillot, A. Julea, L. Valet, J. Chanussot, and M. Koehl. Combining airborne photographs and spaceborne SAR data to monitor temperate glaciers. potentials and limits. IEEE Trans. Geosci. Rem. Sens., 45(4):905-924, 2007.

[2] J.-C. Souyris, C. Henry, and F. Adragna. On the use of complex SAR image spectral analysis for target detection: Assessment of polarimetry. 41(12):2725-2734, 2003.

[3] A. Hooper, H. Zebker, P. Segall, and B. Kampes. A new method for measuring deformation on volcanoes and other natural terrains using InSAR persistent scatterers. Geophysical Research Letters, 31(L23611): 10.1029/2004GL021737, 2004.

[4] G. Vasile, E. Trouvé, J. S. Lee, and V. Buzuloiu. Intensity-drivenadaptive-neighborhood technique for polarimetric and interferometric SAR parameters estimation. IEEE Trans. Geosci. Rem. Sens., 44(5):1609-1621, 2006.

[5] F. Bujor, E. Trouvé, L. Valet, J.M. Nicolas, and J.P. Rudant. Application of log-cumulants to the detection of spatiotemporal discontinuities in multitemporal SAR images. IEEE Trans. Geosci. Rem. Sens., 42(10):2073-2084, october 2004.

[6] G. Vasile, I. Petillot, A. Julea, E. Trouvé, Ph. Bolon, L. Bombrun, M. Gay, T. Landes, P. Grussenmeyer, and J.-M. Nicolas. High resolution SAR interferometry: estimation of local topography in the context of glacier monitoring. In Int. Geosci. and Rem. Sens. Symposium, IGARSS'06, Denver, USA, pages 4008-4011, 2006.

[7] L. Valet, G. Mauris, P. Bolon, and N. Keskes. A fuzzy linguisticbased software tool for seismic image interpretation. IEEE Transactions on Instrumentation and Measurement, 52(3):675-680, 2003.

[8] D. Dubois, L. Foulloy, S. Galichet, and H. Prade. Performing approximate reasoning with words - In Computing with words in Information/Intelligent systems 1. Springer Verlag, 1999.

[9] A. Ferretti, C. Prati, and F. Rocca. Permanent scatterers in SAR interferometry. IEEE Trans. Geosci. Rem. Sens., 39(1):8-20, 2001. 